\title{
Effect of ion concentration on slow light propagation in highly doped erbium fibers
}

\author{
Sonia Melle ${ }^{\mathrm{a}, *}$, Oscar G. Calderón ${ }^{\text {a }}$, F. Carreño ${ }^{\text {a }}$, Eduardo Cabrera ${ }^{\text {a }}$, \\ M.A. Antón ${ }^{a}$, S. Jarabo ${ }^{b}$ \\ a Escuela Universitaria de Óptica, Universidad Complutense de Madrid, Cl Arcos de Jalón s/n, 28037 Madrid, Spain \\ ${ }^{\mathrm{b}}$ Departamento de Física Aplicada, Facultad de Ciencias, Universidad de Zaragoza, Pedro Cerbuna 12, 50009 Zaragoza, Spain
}

Received 4 August 2006; received in revised form 28 June 2007; accepted 30 June 2007

\begin{abstract}
The effect of ion density on slow light propagation enabled by coherent population oscillations has been experimentally investigated for highly doped erbium fibers at room temperature. We found that fractional delay increases with ion density. A saturation effect in the fractional delay has been observed for doping levels above $\sim 3150 \mathrm{ppm}$. Ultra-high ion concentration can simultaneously increase the fractional delay and the bandwidth of the signals. We have studied the propagation of Gaussian pulses along the fibers obtaining fractional delays up to 0.7 for the highest doping levels used. It is shown that pulse power can be used as a control parameter to reduce distortion at different pulse bandwidths.
\end{abstract}

(C) 2007 Elsevier B.V. All rights reserved.

PACS: 42.65.-k; 42.50.Gy

Keywords: Slow-light propagation; Coherence population oscillations; Erbium doped fibers

\section{Introduction}

The process of coherent population oscillations (CPO) allows the reduction of absorption and simultaneously provides a steep spectral variation of the refractive index which leads to a strong reduction of the optical group velocity, i.e., slow light propagation. This process is easily achieved in a two-level system which interacts with a signal whose amplitude is periodically modulated. The population of the ground state of the medium will be induced to oscillate at the modulation frequency. This oscillation creates a narrow hole in the absorption spectrum, whose linewidth is proportional to the inverse of the relaxation lifetime of the excited level [1]. CPO is highly insensitive to dephasing processes in contrast to what happens in other schema such

\footnotetext{
Corresponding author. Tel.: +34 913946909; fax: +34 913946885

E-mail address: smelle@fis.ucm.es (S. Melle).

$U R L:$ http://www.ucm.es/info/laserlab.
}

as electromagnetically induced transparency (EIT) [2-7], where the width of the spectral hole burned in the absorption profile is proportional to the inverse of the dephasing time of the ground state. That makes CPO an appropriate technique to easily achieve slow light propagation in solidstate materials at room temperature.

The first experiment concerning slow light propagation using $\mathrm{CPO}$ at room temperature was carried out by Bigelow et al. [8]. They reported a reduction of the speed of light in a $7.25-\mathrm{cm}-$ long ruby rod down to $57 \mathrm{~m} / \mathrm{s}$ by producing a hole as narrow as $36 \mathrm{~Hz}$ (HWHM) in the absorption spectrum. Thus, pulses of $25 \mathrm{~ms}$ width (FWHM) were delayed by $0.71 \mathrm{~ms}$ and fractional delays of 0.028 were reached. Later, other materials were shown to successfully produce slow-light propagation based on $\mathrm{CPO}$, as in alexandrite crystals [9] and in semiconductor structures [10-12]. Furthermore, using cryogenic temperatures, a decrease of group velocity down to $3 \mathrm{~m} / \mathrm{s}$ was recently reported in a 3-mm-long erbium-doped crystal [13]. Thanks to the long 
lifetime of the excited state of the Er ions, a spectral hole as narrow as $26 \mathrm{~Hz}$ (HWHM) is produced, thus providing fractional delays of the order of 0.012 .

An interesting work by Schweinsberg et al. [14] has shown slow light propagation of both sinusoidally modulated signals and Gaussian pulses in an erbium-doped fiber (EDF) at the telecommunication wavelength of $1.55 \mu \mathrm{m}$. They used a $13 \mathrm{~m}$-long EDF with an Er ion density of $1.78 \times 10^{24} \mathrm{~m}^{-3}(90 \mathrm{ppm})$. Pulses as short as $3.2 \mathrm{~ms}$-wide (FWHM) were delayed by $0.38 \mathrm{~ms}$, hence fractional delays of 0.12 were achieved. The use of optical fibers to produce slow-light propagation has many advantages over other solid-sate systems since they operate at the telecommunication window, allowing for the use of long interaction lengths. Thus, EDFs are promising candidates for controllable optical delay lines and optical buffers.

The important issue in slow-light delay lines is not merely the achievement of large time delays, but to produce large fractional delays (time delay normalized to the time duration of the signal) since this parameter roughly measures the information storage capacity of the optical medium [15]. Moreover, most practical applications require that large modulation bandwidths be able to experience slow-light effects [15]. Thus, large fractional delays and large modulation bandwidths are desirable simultaneously. Many applications require a minimization of fiber length without the reduction of achievable fractional delay [15]. A possible way to increase the fractional delay is to use fiber with a high ion doping level. In this work we study the effect of ion density on slow-light propagation. We used EDFs with ion concentrations ranging from 10 to 50 times greater than that used in previous work [14]. We analyzed how ion density influences the fractional delay and bandwidth. We found that ultra-highly doped erbium fibers show simultaneously higher bandwidths and larger fractional delays than do highly doped fibers.

\section{Experimental setup}

The experimental setup is depicted in Fig. 1. We use a distributed feedback laser diode (Anritsu, model GB5A016) operating at $1536.2 \pm 0.5 \mathrm{~nm}$. The laser power is controlled with an accuracy of $0.01 \mathrm{~mW}$ using a combined laser and temperature controller (Thorlabs, model ITC502) that allows us to keep the laser at room temperature with a stability of $0.005^{\circ} \mathrm{C}$. The laser output is split into two signals: one part of the laser signal $(99 \%)$ goes through an EDF $1 \mathrm{~m}$ in length and then to a switchablegain, amplified InGaAs photodetector (Thorlabs, model PDA400) with $10 \mathrm{MHz}$ bandwidth at the minimum gain setting. The other part of the laser output signal (1\%) is sent directly to an identical photodetector to be used as reference. An in-line variable optical attenuator (Thorlabs, model VOA50) was placed between the EDF and the detector to prevent saturation of the photodetector. Both the reference signal and the EDF signal are recorded with a fast data acquisition card (rate of $10^{7}$ samples/s) (National

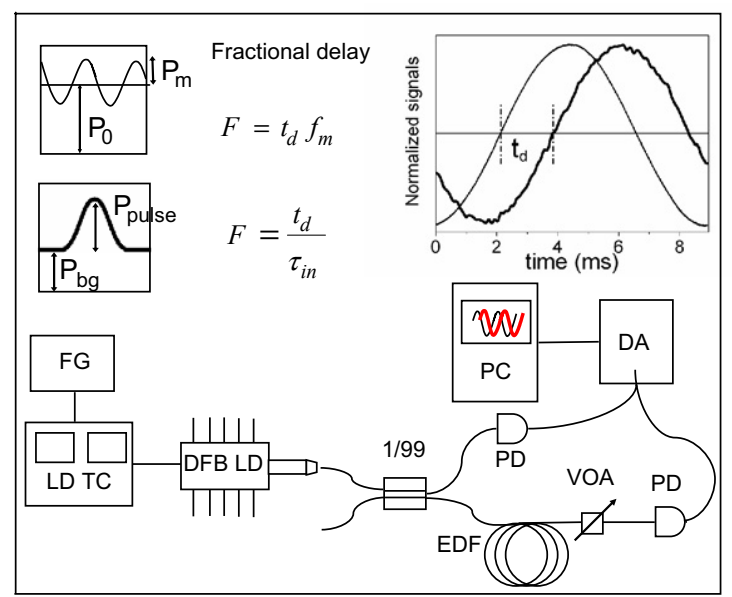

Fig. 1. Experimental setup used to measure slow light in erbium-doped fibers. LD TC, laser diode and temperature controller; FG, function generator; DFB LD, distributed feedback laser diode; EDF, erbiumdoped fiber; VOA, variable optical attenuator; PD, photodetector; DA, data acquisition card; PC, personal computer. The upper-right corner shows the normalized modulated reference signal at $f_{\mathrm{m}}=120 \mathrm{~Hz}$ (thin line) and the corresponding EDF signal (thick line) after propagating through the Er80 fiber. The laser input power was $P_{0}=0.13 \mathrm{~mW}$ and the modulation amplitude was $P_{\mathrm{m}}=0.065 \mathrm{~mW}$. The signal was delayed $t_{\mathrm{d}}=1.7 \mathrm{~ms}$ corresponding to an average group velocity of $v_{\mathrm{g}} \sim 580 \mathrm{~m} / \mathrm{s}$, i.e. a group index of $n_{\mathrm{g}} \sim 5 \times 10^{5}$.

Instruments, model PCI-6115 S series) and sent to a computer for analysis. The experiment is controlled with a LabView program.

We use single mode, $\mathrm{Al}_{2} \mathrm{SiO}_{5}$-glass-based fibers doped with $\mathrm{Er}^{3+}$ ions at several concentrations (provided by Liekki Ltd.) The transition between the ground state ${ }^{4} I_{15 / 2}$ and the excited metastable state ${ }^{4} I_{13 / 2}$ of the erbium ions is at $1536 \mathrm{~nm}$. The ion density corresponding to each fiber, shown in Table 1, ranges from $800 \mathrm{ppm}$ to $4350 \mathrm{ppm}$. In what follows we will refer to the differently doped fibers with a code associated to their nominal peak absorption according to Table 1 . All the fibers have a nominal mode field diameter at $1550 \mathrm{~nm}$ of $6.5 \mu \mathrm{m}$, a fiber cladding of $245 \mu \mathrm{m}$, and a numerical aperture of 0.20 .

We have carried out two kinds of experiments. In the first, the injection current of the laser was sinusoidally modulated by a function generator (Agilent, model $33220 \mathrm{~A}$ ) so that the laser power injected into the fiber was $P=P_{0}+P_{\mathrm{m}} \cos \left(2 \pi f_{\mathrm{m}} t\right)$, where $P_{0}$ is the average power, $P_{\mathrm{m}}$ the modulation amplitude, and $f_{\mathrm{m}}$ the modulation frequency. We computed the time delay $t_{\mathrm{d}}$ from the

Table 1

Properties of the EDFs

\begin{tabular}{lclc}
\hline $\begin{array}{l}\text { Fiber } \\
\text { code }\end{array}$ & $\begin{array}{l}\text { Peak absorption } \\
(\mathrm{dB} / \mathrm{m})\end{array}$ & $\begin{array}{l}\text { Ion density } \rho \\
\left(\mathrm{m}^{-3}\right)\end{array}$ & $\begin{array}{l}\text { Ion concentration } \\
(\mathrm{ppm})\end{array}$ \\
\hline Er20 & $20 \pm 2$ & $1.6 \times 10^{25}$ & 800 \\
Er30 & $30 \pm 3$ & $2.1 \times 10^{25}$ & 1050 \\
Er40 & $40 \pm 4$ & $2.7 \times 10^{25}$ & 1350 \\
Er80 & $80 \pm 8$ & $6.3 \times 10^{25}$ & 3150 \\
Er110 & $110 \pm 10$ & $8.7 \times 10^{25}$ & 4350 \\
\hline
\end{tabular}


correlation between the reference signal and the EDF signal. The fractional delay is then defined as $F=t_{\mathrm{d}} f_{\mathrm{m}}$. In the second experiment, we considered the propagation of Gaussian-shaped optical pulses produced by the function generator over a laser power background in such a way that the laser power in this case was $P=P_{\mathrm{bg}}+$ $P_{\text {pulse }} \cdot \exp \left(-t^{2} / 2 \sigma_{\text {in }}^{2}\right)$, where $P_{\text {bg }}$ and $P_{\text {pulse }}$ are the background and pulse power, respectively, and $\sigma_{\text {in }}^{2}$ is the variance of the distribution of the Gaussian input pulse. Thus we define $\tau_{\text {in }}$, the full-width at half the maximum of the input pulse, as $\tau_{\text {in }} \equiv 2 \sqrt{2 \ln 2} \sigma_{\text {in }}$. In this case, the fractional delay is obtained from $F=t_{\mathrm{d}} / \tau_{\mathrm{in}}$. In all the experiments reported here, a laser background power of $P_{\mathrm{bg}}=0.06 \mathrm{~mW}$ was used.

\section{CPO model}

The propagation of an amplitude periodically-modulated signal through an erbium-doped fiber has been modeled in previous works of $\mathrm{CPO}$ using a rate equation analysis $[8,13,14]$. Following those works, we consider this model to study the interaction between power-modulated $1536 \mathrm{~nm}$ light and the two-level atomic system of the erbium ions. The propagation equations of the signal power $P$ and the population $N_{1}$ of the ground state ${ }^{4} I_{15 / 2}$ are

$$
\begin{aligned}
& \frac{\mathrm{d} P}{\mathrm{~d} z}=P \sigma_{21} N_{2}-P \sigma_{12} N_{1}, \\
& \frac{\mathrm{d} N_{1}}{\mathrm{~d} t}=\frac{N_{2}}{\tau}+\frac{P \sigma_{21}}{\hbar \omega A} N_{2}-\frac{P \sigma_{12}}{\hbar \omega A} N_{1},
\end{aligned}
$$

where $z$ is the distance along the fiber and $N_{2}$ is the population of the excited metastable state ${ }^{4} I_{13 / 2}$. Here $\sigma_{21}$ and $\sigma_{12}$ are the emission and absorption cross sections, respectively. $\tau$ is the lifetime of the metastable state, $A$ is the mode field area, and $\omega$ is the transition frequency between the ground and excited states of the erbium ions $\left({ }^{4} I_{15 / 2} \rightarrow\right.$ $\left.{ }^{4} I_{13 / 2}\right)$. Due to the modulation of the signal $P=$ $P_{0}+P_{\mathrm{m}} \cos \left(2 \pi f_{\mathrm{m}} t\right)$ a periodic coherent oscillation of the population of the metastable state occurs, producing a hole in the absorption spectrum and leading to slow light propagation. The propagation equation of the average power signal $P_{0}$ through the fiber reads (see Ref. [8])

$\frac{\partial \widehat{P}_{0}}{\partial z}=-\frac{\alpha_{0} \widehat{P}_{0}}{1+\widehat{P}_{0}}$.

$\hat{P}_{0}=P_{0} / P_{\text {sat }}$ is the ratio of the average laser power $P_{0}$ to the saturation power $P_{\text {sat }}=\hbar \omega A /\left(\tau\left(\sigma_{21}+\sigma_{12}\right)\right)$, and $\alpha_{0}=\sigma_{12} \rho$ is the unsaturated absorption coefficient of the two-level system, $\rho$ being the ion density. The phase delay experienced by the periodic part of the signal due to CPO is given by the following propagation equation (see Ref. [14])

$\frac{\partial \phi}{\partial z}=\alpha_{0} \frac{\widehat{P}_{0}}{1+\widehat{P}_{0}} \frac{2 \pi f_{\mathrm{m}} \tau}{\left(1+\hat{P}_{0}\right)^{2}+\left(2 \pi f_{\mathrm{m}} \tau\right)^{2}}$.

The fractional delay can be obtained from $F=\phi /(2 \pi)$. Analytical expressions have been deduced in Refs.
$[8,9,13,14]$ by neglecting the attenuation of the laser signal. This approximation has not been assumed in our work due to the high absorption of the highly doped fibers used, a point to be addressed in the next section. Analytical results are shown here for purposes of comparison. Hence, it can be easily seen analytically that the maximum fractional delay $F_{\max }$ is achieved at the modulation frequency $f_{\mathrm{m}}=f_{\mathrm{opt}}$ $[8,13,14]$ :

$f_{\text {opt }}=\frac{1}{2 \pi \tau}\left(1+\hat{P}_{0}\right)$.

The value of this optimum modulation frequency corresponds to the HWHM of the spectral hole induced by $\mathrm{CPO}$ effects in the absorption spectrum and gives us an idea of the operating bandwidth of the system. The value of the maximum fractional delay $F_{\max }$ is given by

$F_{\max }=\frac{\alpha_{0} L}{4 \pi} \frac{\widehat{P}_{0}}{\left(1+\hat{P}_{0}\right)^{2}}$

where $L$ is the fiber length. From these analytical results it would be expected that the bandwidth of the system does not change with ion density, since under this approximation $f_{\text {opt }}$ is the same for all the fibers (see Eq. (5)). Furthermore, the maximum fractional delay would be expected to increase linearly with the unsaturated absorption coefficient, and therefore with ion density (see Eq. (6)).

\section{Experimental results and discussion}

We have characterized the transmission of a $\mathrm{CW}$ signal through the fibers. To this end we measured the input-output power curve for the different fibers. As an example, Fig. 2 shows the input-output power curve for the Er20 fiber. In the experiments reported here, the input laser power $P_{0}(z=0)$ was varied from $0.04 \mathrm{~mW}$ to $6.5 \mathrm{~mW}$. A saturation regime, defined by a linear relation of slope unity between the input and output power, is reached for

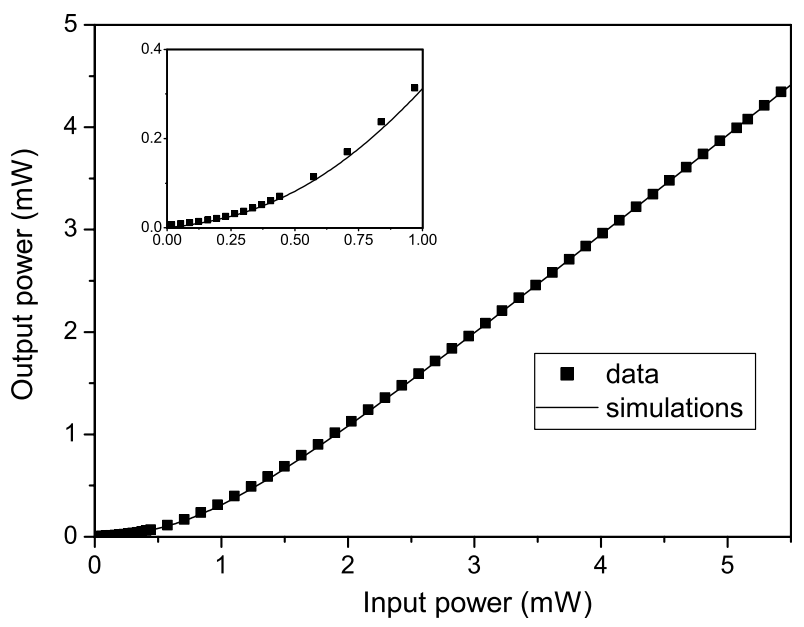

Fig. 2. Experimental (symbols) and simulated (solid line) input-output power curves for the Er20 fiber. The inset displays the input-output curves for the low power regime. 
fibers Er20, Er30 and Er40 at input powers greater than $1.5 \mathrm{~mW}, 2 \mathrm{~mW}$ and $3 \mathrm{~mW}$, respectively. However, for fibers Er80 and Er110, we observed that the saturation regime is not reached even for the greatest input laser power used in the experiments (not shown). In particular, for $P_{0}(z=0)=6.5 \mathrm{~mW}$, the measured output power is $P_{0}(z=L)=130 \mu \mathrm{W}$ for Er80 fiber and $P_{0}(z=L)=8 \mu \mathrm{W}$ for Er110 fiber. These results clearly indicate a strong attenuation of the signals propagating through the ultrahighly doped erbium fibers (Er80 and Er110). Therefore, it would be expected that the behavior of the optimum frequency $f_{\text {opt }}$ and the maximum fractional delay $F_{\text {max }}$ should not follow the analytical expressions given by Eqs. (5) and (6). In view of this, we resort to determining the spatial evolution of $P_{0}$ and $\phi$ by numerical methods.

By numerically solving Eq. (3) we calculate the inputoutput power curve considering similar absorption and emission cross sections $\sigma_{12} \simeq \sigma_{21}=\sigma$. The numerical results are fitted to the experimental data using the absorption cross section $\sigma$ and the effective mode field diameter $\mathrm{d}$ as variable parameters. The fitting provides a value of $\sigma=1.8 \times 10^{-25} \mathrm{~m}^{2}$ (consistent with values previously used in other works $[14,16]$ ) and a value of $\mathrm{d}=3.9 \mu \mathrm{m}$ (close to the value given by the manufacturer). Thus, the saturation intensity is $I_{\mathrm{sat}} \simeq 3.4 \mathrm{~kW} / \mathrm{cm}^{2}\left(P_{\mathrm{sat}} \simeq 0.4 \mathrm{~mW}\right)$ and the unsaturated absorption coefficient in our experiments ranges from $\alpha_{0}=0.03 \mathrm{~cm}^{-1}$ to $\alpha_{0}=0.16 \mathrm{~cm}^{-1}$ (see Table 1 for the corresponding values of $\rho$ ). The metastable state lifetime we considered is $\tau=10.5 \mathrm{~ms}$ [14]. In Fig. 2 (solid line) we show the simulated input-output power curve obtained for the Er20 using the above mentioned parameters.

\subsection{Sinusoidally modulated signal}

For each fiber we have measured the fractional delay $F$ as a function of the modulation frequency $f_{\mathrm{m}}$. In order to analyze the influence of the modulation amplitude $\left(P_{\mathrm{m}}\right)$ on the fractional delay, we changed the ratio of modulation amplitude to average input power from 10 to $70 \%$, thereby changing $P_{\mathrm{m}} / P_{0}$ from 0.1 to 0.7 . As an example, Fig. 3 displays the experimental fractional delay for different modulation amplitudes as a function of the modulation frequency in a semilog scale. Those measurements were carried out at a fixed average input laser power of $P_{0}=0.6 \mathrm{~mW}$. The figure on the left shows the results for the least highly doped fiber $(\operatorname{Er} 20)$, and the one on the right shows the results for the most highly doped fiber (Er110). Note that, in both cases, the fractional delay does not exhibit significant changes upon the change of modulation amplitude. Thus, in what follows we consider a modulation amplitude of $50 \%$. A further inspection of Fig. 3 reveals that the maximum fractional delay $F_{\max }$ and the optimum modulation frequency at which this maximum fraction delay occurs $\left(f_{\text {opt }}\right)$ are both very different for each fiber. In particular, for the least highly doped fiber (Er20, on the left) the maximum fractional delay achieved is $F_{\max } \approx 0.05$, whereas for the most highly doped fiber (Er110, on the right) the maximum fractional delay increases by a factor of 3.6 to $F_{\max } \approx 0.18$. This increase factor is smaller than the ratio between ion concentrations and could indicate that $F_{\max }$ is not proportional to ion density. Furthermore, the optimum frequency at which the maximum fractional delay takes place appears with a value around $f_{\text {opt }} \approx 30 \mathrm{~Hz}$ for the Er20 doped fiber (see Fig. 3 left) and shifts to a higher frequency value around $f_{\text {opt }} \approx 150 \mathrm{~Hz}$ for the Er110 fiber (see Fig. 3 right). Thus, the bandwidth of the system exhibits a strong dependence upon ion density, which is not evident in the simple model used previously (Eq. (5)).

In order to analyze the role of ion density in the slowdown of light in EDFs, we measured the fractional delay as a function of the modulation frequency for a laser power of $P_{0}=0.6 \mathrm{~mW}$ and for fibers with different doping levels. The results have been plotted in Fig. 4a. Note that, as ion density increases from $800 \mathrm{ppm}$ (Er20) to $4350 \mathrm{ppm}$ (Er110), fractional delay increases as well. The dependence of the maximum fractional delay $F_{\max }$ upon ion density can

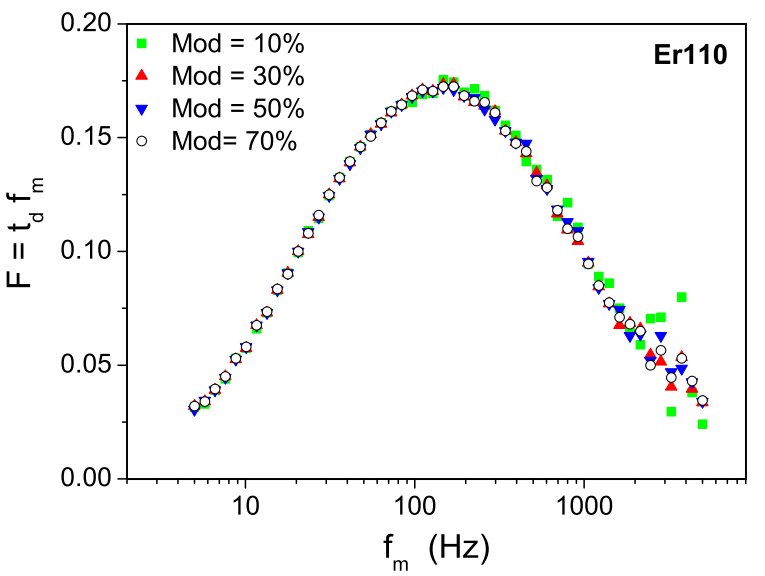

Fig. 3. Fractional delay as a function of the modulation frequency at a laser power of $0.6 \mathrm{~mW}$, with different modulation amplitudes regarding the input laser power $\left(\mathrm{Mod}=100 \times P_{\mathrm{m}} / P_{0}\right)$. On the left Er20 fiber; on the right Er110 fiber. 

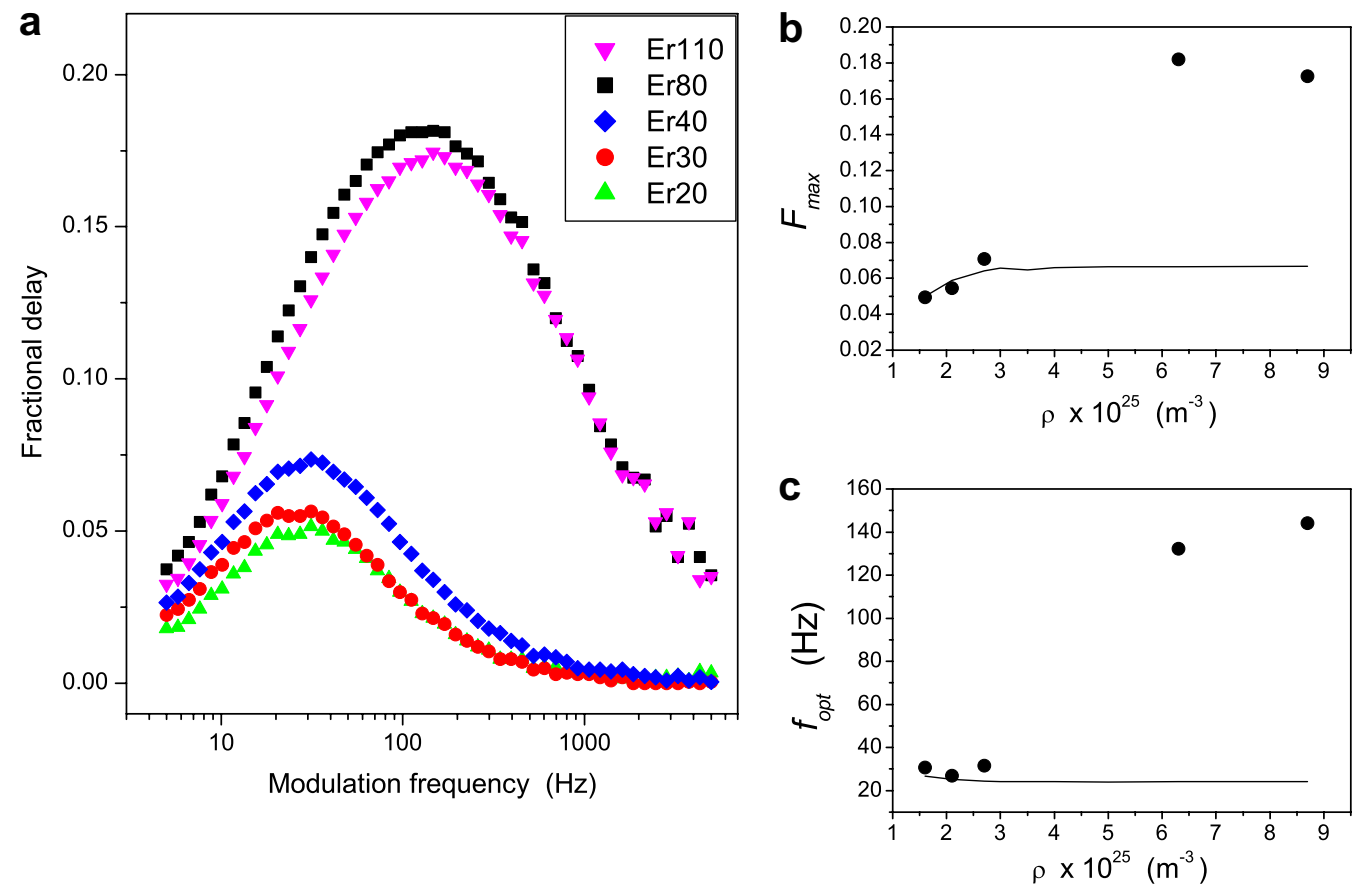

Fig. 4. (a) Fractional delay as a function of the modulation frequency for a laser power of $0.6 \mathrm{~mW}$ and for fibers with different $\mathrm{Er}^{3+}$ ion concentration. (b) Maximum fractional delay $F_{\max }$ as a function of ion density $\rho$. (c) Optimum modulation frequency $f_{\text {opt }}$ as a function of ion density $\rho$. The solid lines in Figs. (b) and (c) correspond to the simulation results obtained by numerically solving Eqs. (3) and (4).

be seen in Fig. 4b. A linear increase is found for ion densities below $3 \times 10^{25} \mathrm{~m}^{-3}$, in agreement with the numerical results obtained by solving Eqs. (3) and (4) (see solid line). We observed a saturation of the maximum fractional delay achieved with high ion densities (corresponding to fibers Er80 and Er110). The model also predicts such a saturation, although the predicted values are far from the experimental data for Er80 and Er110 fibers.

The optimum frequency at which the maximum fractional delay occurs appears at a fixed value around $f_{\text {opt }} \approx 30 \mathrm{~Hz}$ for the Er20-Er40 fibers (see Fig. 4c). This value agrees with those calculated via simulations (see solid line). However, for more highly doped fibers (Er80 and Er110) this optimum frequency shifts to higher frequencies (not reproduced by the simulations). This means that ultrahighly doped fibers show large bandwidths with high fractional delays as previously pointed out in connection with Fig. 3 (right). In particular, the transparency window for the Er110 fiber exhibits a bandwidth as large as $150 \mathrm{~Hz}$, compared to the $31 \mathrm{~Hz}$ found for the Er20 fiber.

In order to clarify the different behavior found for the optimum frequency $f_{\text {opt }}$ in Fig. $4 \mathrm{c}$ and for the maximum fractional delay $F_{\max }$ in Fig. 4 b, for each fiber we study the dependence of $f_{\text {opt }}$ and $F_{\text {max }}$ upon laser input power. As an example we show representative results of both groups of fibers: Er20 for highly EDFs, and Er110 for ultra-highly EDFs. Fig. 5 shows the fractional delay versus modulation frequency for the Er20 fiber and for different
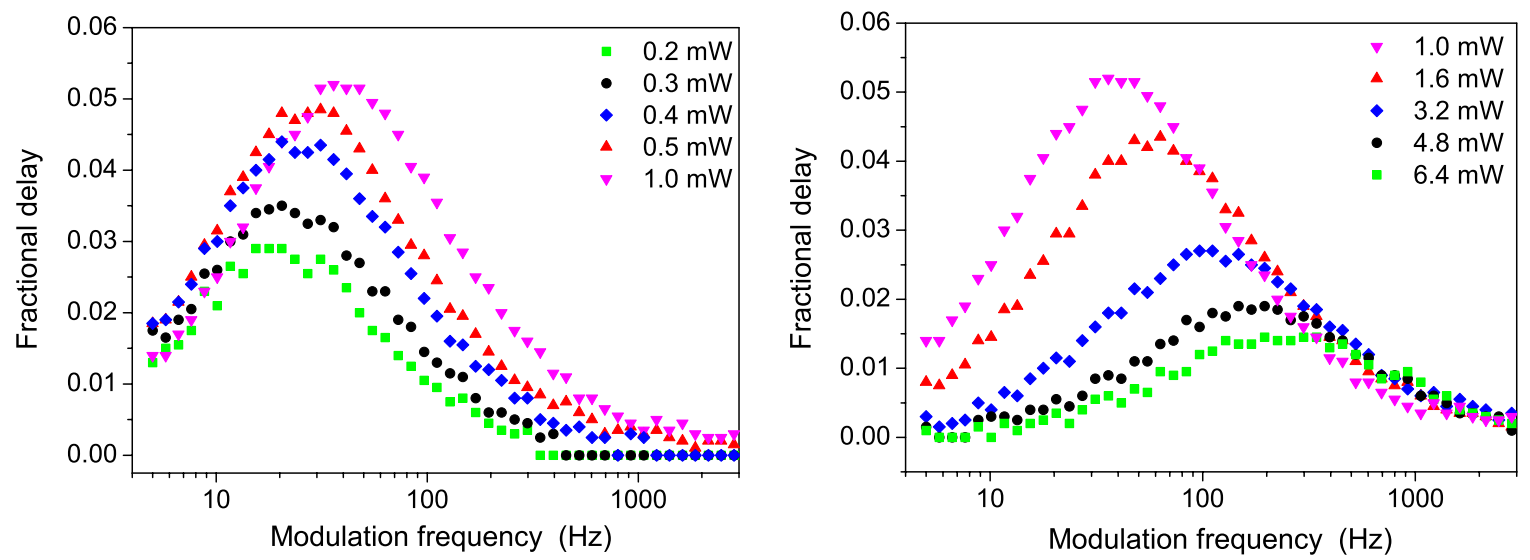

Fig. 5. Fractional delay as a function of the modulation frequency for fiber $\operatorname{Er} 20\left(\rho=1.6 \times 10^{25} \mathrm{~m}^{-3}\right)$ and different laser powers. Figure has been divided into two plots for clarity: (left) low laser input powers; (right) high laser input powers. 
laser input powers $P_{0}$; Fig. 5 has been divided into two plots for the sake of clarity. We observed an increase in the optimum modulation frequency with the laser power from $21 \mathrm{~Hz}$ (for $P_{0}=0.2 \mathrm{~mW}$ ) to $232 \mathrm{~Hz}$ (for $P_{0}=6.4 \mathrm{~mW}$ ). This increase is more pronounced when surpassing a laser power around $P_{0} \simeq 1 \mathrm{~mW}$ (see right part of Fig. 5). We can also see in Fig. 5 (on the left) that the fractional delay increases upon increasing laser power until a laser power around $P_{0} \simeq 1 \mathrm{~mW}$ is reached. For laser powers above $P_{0} \simeq 1 \mathrm{~mW}$, the fractional delay decreases upon increasing laser power (see right plot of Fig. 5). This decrease is an expected result since, because we are working above the saturation intensity through most of the fiber length, the number of excited ions contributing to the $\mathrm{CPO}$ effect is reduced as a consequence of saturation. This saturation leads to a power broadening of the spectral hole which produces an increase of group velocity [13].

In Fig. 6 we present the corresponding results for the Er110 fiber. Note that for low laser powers (on the left) the optimum modulation frequency increases abruptly with laser power. However, contrary to the behavior found for the Er20 fiber, the bandwidth for this fiber remains unaltered upon raising laser power above $P_{0}=0.13 \mathrm{~mW}$. Furthermore, the maximum fractional delay increases abruptly up to a value of $F_{\max } \approx 0.2$, reached when the laser input power is $P_{0}=0.13 \mathrm{~mW}$. Above this power value the maximum fractional delay decreases very slowly upon increasing laser input power. This is a great advantage over the less-doped fibers, for in these fibers it is not necessary to increase the laser power in order to obtain larger bandwidths.

In order to summarize our results, we plot in Fig. 7 the experimental data (in symbols) for $f_{\text {opt }}$ and $F_{\max }$ as a function of laser power, together with the simulated values obtained by numerically solving Eqs. (3) and (4) (solid line). As we mentioned before, the group of fibers with low ion concentration (Er20, Er30 and Er40) behaves differently from the group of ultra-highly doped fibers (Er80 and Er110). For the least highly doped fibers (Er20, and Er30) the optimum modulation frequency does not change
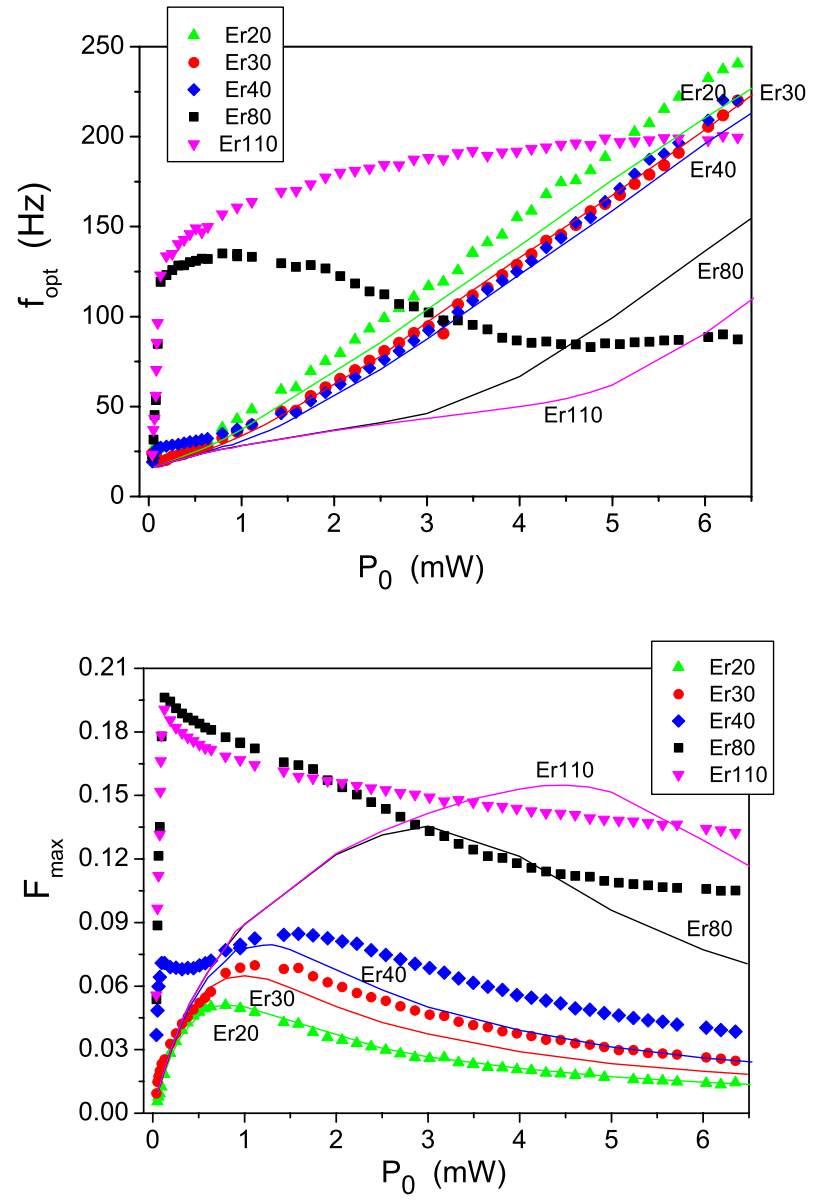

Fig. 7. Optimum modulation frequency (up) and maximum fractional delay (down) as a function of input laser power for fibers of different ion concentration: Experimental results (symbols), and simulated result from numerically solving Eqs. (3) and (4) (line). Each line is labeled with its corresponding fiber.

appreciably when the input laser power is smaller than $P_{0} \approx 1 \mathrm{~mW}$. These low input powers are not enough to broaden the hole in the absorption spectra and therefore the hole width only depends upon the lifetime of the metastable state $\tau$. However, for laser powers greater than the
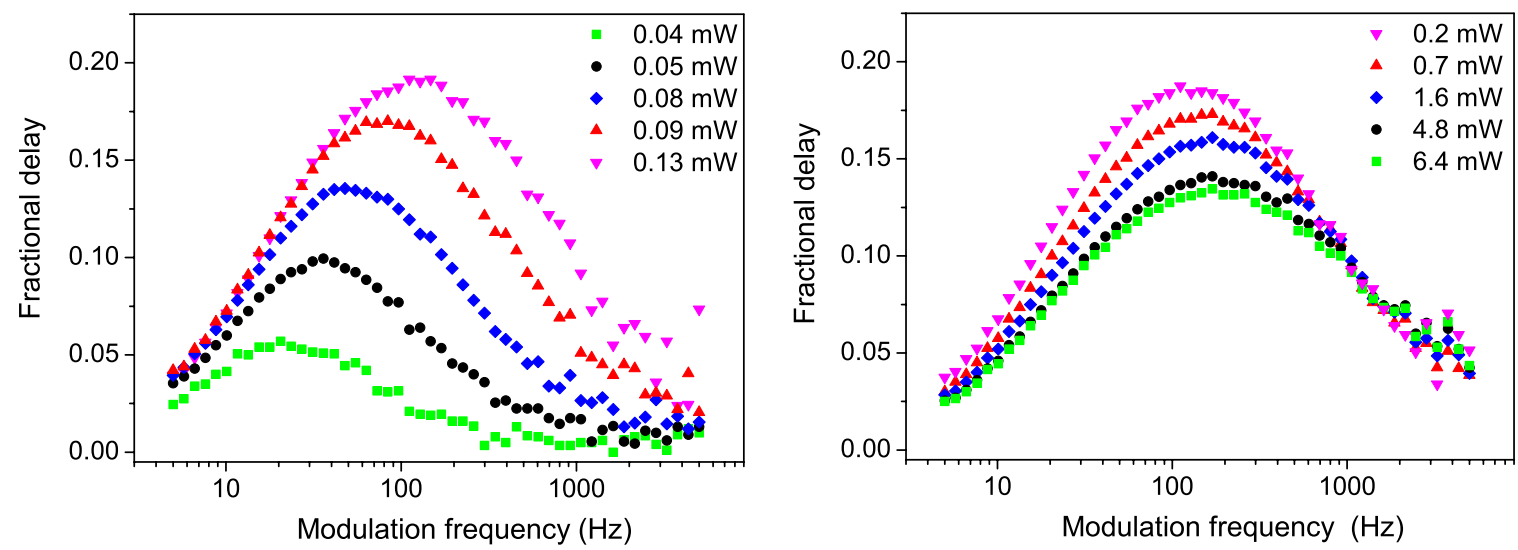

Fig. 6. Fractional delay as a function of the modulation frequency for fiber $\operatorname{Er} 110\left(\rho=8.7 \times 10^{25} \mathrm{~m}^{-3}\right)$ and different laser powers. Figure has been divided into two plots for clarity: (left) low laser input powers; (right) high laser input powers. 
power that saturates the whole fiber $\left(P_{0}>1 \mathrm{~mW}\right)$, a broadening of the spectral hole leads to a linear increase of $f_{\text {opt }}$ with the input laser power. This behavior has been reproduced by the simulations (see solid line in Fig. 7). In the case of ultra-highly doped fibers (Er80 and Er110) an abrupt increase of $f_{\text {opt }}$ for laser powers below $P_{0}=0.13 \mathrm{~mW}$ can be clearly seen. For laser input powers greater than this value a saturation of the hole width can be observed. This behavior cannot be reproduced by the simulations (see line in Fig. 7).

In Fig. 7 (down) we see that in the case of low doped fibers (Er20 and Er30) the maximum fractional delay grows upon increasing the input laser power until a maximum value around 0.05 is reached (at $P_{0} \approx 1 \mathrm{~mW}$ ), above which the $F_{\max }$ decreases slowly with laser power. As we said before, an increase of laser power produces a more pronounced dip in the absorption spectrum and the corresponding increment of $F_{\max }$. However, if we keep increasing the laser power above saturation a broadening of the spectral hole occurs so that the fractional delay decreases. This slow decrease of the maximum fractional delay above the saturation power is also observed for the Er40 fiber. However, this fiber shows a steep increase for very low laser powers $\left(P_{0} \leqslant 0.13 \mathrm{~mW}\right)$ that distorts the smooth growth predicted by the simulations (see solid line for Er40 fiber in Fig. 7 (down)). In the case of ultra-highly doped fibers (Er80 and Er110) the abrupt growth of the maximum fractional delay for laser powers below $P_{0}=0.13 \mathrm{~mW}$ is more pronounced. Moreover, a saturation effect can be seen since the maximum fractional delay for both fibers (Er80 and Er110) is almost the same, around $F_{\max } \approx 0.2$. Thus, if we want to optimize the fractional delay while having maximum transmission, it will be more efficient to use Er80 fiber than Er110 fiber. So an ion concentration closer to $\sim 3000 \mathrm{ppm}$ will be more appropriate to get the maximum fractional delay while obtaining a moderate transmitted signal. From the above results we can conclude that ultra-high ion concentrations can simultaneously increase the fractional delay and the bandwidth of the slow light effect even in the low power regime.

Note that the anomalous behaviors found for the ultrahighly EDFs are not predicted by the simulations: (i) the saturation of the fractional delay with increased ion concentration, (ii) the unchangeable bandwidth with laser powers above $P_{0} \simeq 0.13 \mathrm{~mW}$, and (iii) the strong increase in the magnitude of both $f_{\text {opt }}$ and $F_{\text {max }}$ for laser powers below $P_{0} \simeq 0.13 \mathrm{~mW}$. These facts reveal that the simple model derived from Eqs. (3) and (4) must be reconsidered and that other physical phenomenon need to be included in the model in order to properly reproduce the experimental findings. Note also that the unexpected behavior that appears for ultra-highly EDFs may serve as an indication that interparticle interaction effects may play an important role in the slow-down of light at these levels of ion doping. In particular, cooperative upconversion effects have been suggested as a phenomenon contributing to fiber dynamics [17-20]. We have taken into account the cooperative upconversion among uniformly distributed erbium ions by adding to the rate equation of the population of the ground state $N_{1}$ (given by Eq. (2)) a quadratic term of the form $K N_{2}^{2}$. This term represents the process in which one excited erbium ion (donor) donates its energy to a neighbor excited erbium ion (acceptor). As a result of this interaction, one excited ion is lost. In other words, cooperative upconversion may help to increase the bandwidth of the slow light effect by speeding the removal of population from the excited state. We have solved Eqs. (1) and (2) by adding a new term which takes into account the uniform upconversion effect. We have considered a value of the parameter $K$ between $K=10^{-22} \mathrm{~m}^{3} \mathrm{~s}^{-1}$ and $K=10^{-24}$ $\mathrm{m}^{3} \mathrm{~s}^{-1}$ as has been previously used in literature [17$20,16]$. The results obtained from the numerical simulations show no significant improvement in the fitting to the experimental data, as can be seen in Fig. 8. Note that the behavior of the optimum frequency $f_{\text {opt }}$ is dramatically changed for large values of the upconversion parameter. In particular, a fast increase takes place at low input powers in
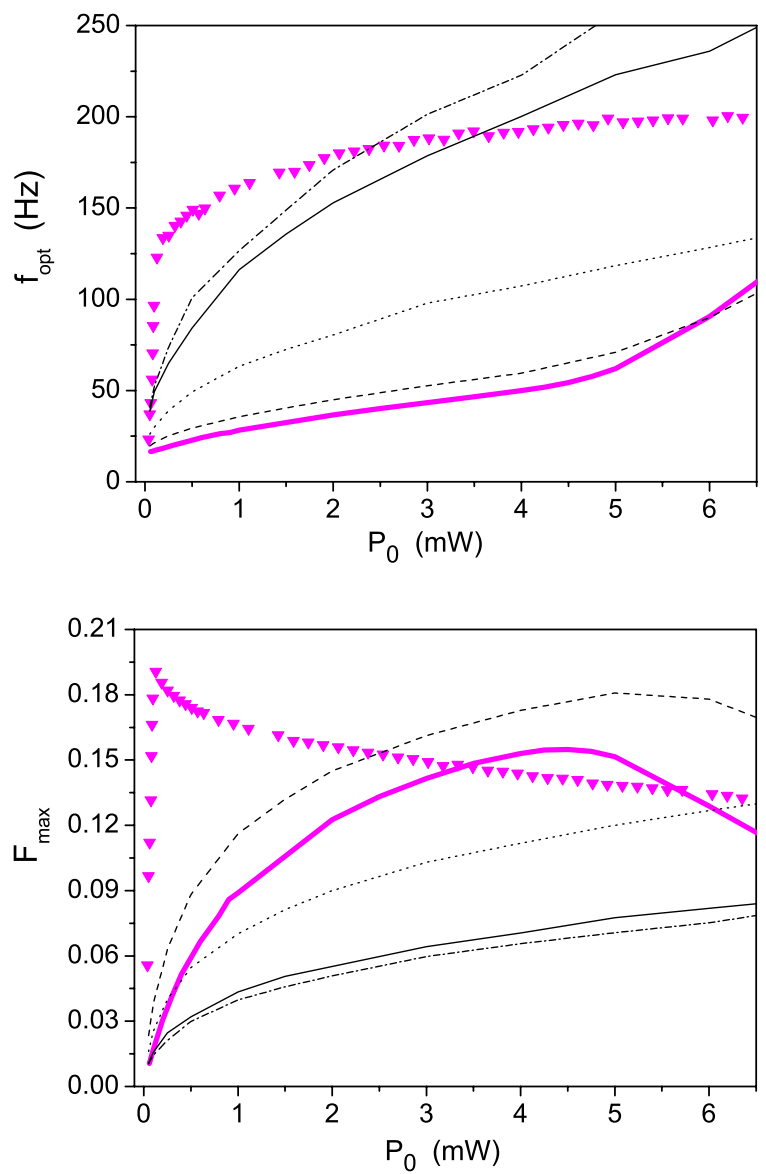

Fig. 8. Optimum modulation frequency (up) and maximum fractional delay (down) as a function of input laser power for the highest doped fiber (Er110). Experimental results (symbols), and simulated result from numerically solving Eqs. (3) and (4) (thick line). The other curves have been obtained by taking into account the uniform upconversion effect with different upconversion parameter: $K=1 \times 10^{-24} \mathrm{~m}^{3} / \mathrm{s}$ (dashed line), $K=10 \times 10^{-24} \mathrm{~m}^{3} / \mathrm{s}$ (dotted line), $K=40 \times 10^{-24} \mathrm{~m}^{3} / \mathrm{s}$ (solid line), and $K=50 \times 10^{-24} \mathrm{~m}^{3} / \mathrm{s}$ (dashed-dotted line). 
agreement with the experiments. However, the behavior of the maximum fractional delay at low input powers is not qualitatively reproduced by the simulations. These results point out that other phenomena should be taken into account. Wysocki et al. [16] analyzed interparticle interactions to explain the effect of ion concentration in the erbium-doped fiber amplifier efficiency. They found that uniform cooperative upconversion fails to explain the effect of ion concentration. However, pair interaction was found to be the mechanism which explains their results. It seems, then, that more complex numerical models, including the rapid cross-relaxation between paired ions, should be used to reproduce the behavior of ultra-highly doped erbium fibers. Further studies are currently being carried out to address this matter.

\subsection{Gaussian pulse signal}

We have studied how finite-band signals propagate through EDFs. The shape of the pulses was selected to be Gaussian over a DC component whose power is $P_{\mathrm{bg}}$. In the top portion of Fig. 9 we plot a reference Gaussian pulse of FWHM $\tau_{\text {in }}=33 \mathrm{~ms}$ (dotted line) and the corresponding delayed pulse after propagating through two different Er doped fibers (solid line): Er20 (on the left) and
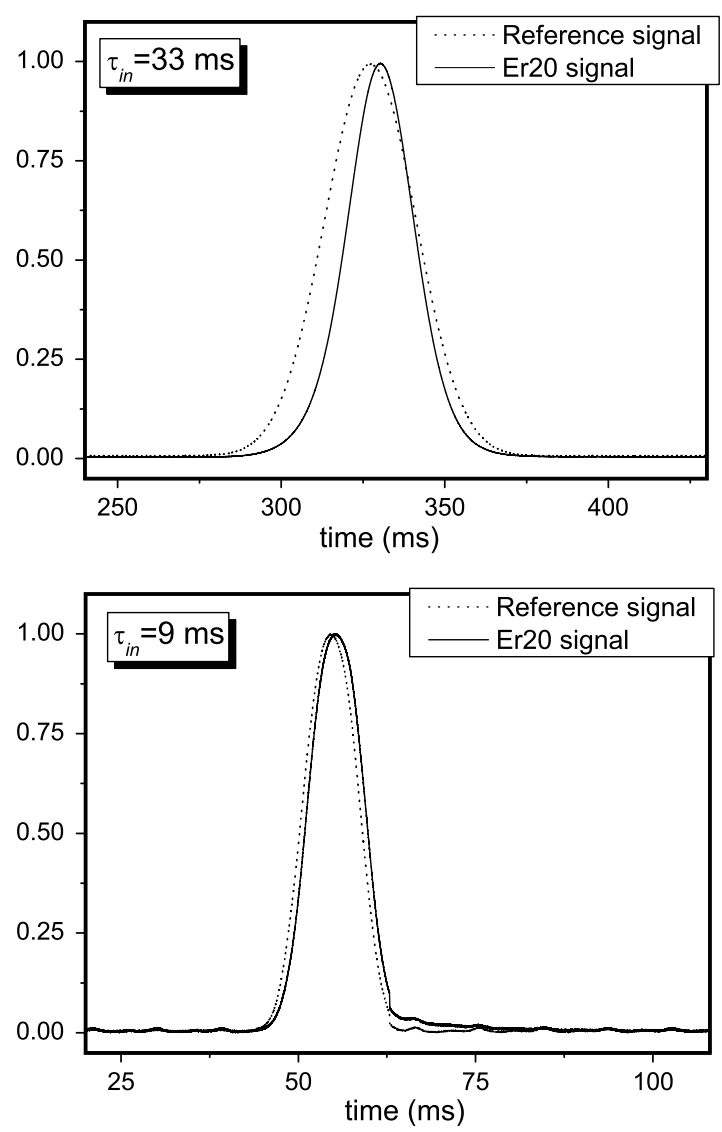

Er110 (on the right). The laser background power was set to $P_{\mathrm{bg}}=0.06 \mathrm{~mW}$ (below the saturation power), and the pulse-to-background power ratio was $P_{\text {pulse }} / P_{\mathrm{bg}}=25$ for the pulse propagating along the Er20 fiber and $P_{\text {pulse }} /$ $P_{\mathrm{bg}}=29$ for the one propagating along the Er110 fiber. The time delay for the pulse propagated through the Er20 fiber $(3.08 \mathrm{~ms})$ is smaller than the time delay for the one propagated through the Er110 fiber $(6 \mathrm{~ms})$. This result is in accordance with the results obtained in the case of sinusoidally modulated signals. Thus, the fractional delay obtained for the Er110 fiber $(F=0.18$, which corresponds to a group velocity of $167 \mathrm{~m} / \mathrm{s}$ ) is approximately twice the value of the fractional delay obtained for the Er20 fiber $(F=0.09)$. Furthermore, we can see at the top of Fig. 9 that the pulse propagated through the Er20 fiber is shorter in width than the input pulse (FWHM $\tau_{\text {out }}=23.7 \mathrm{~ms}$ ), while the pulse propagated through the Er110 fiber is larger in width than the input pulse (FWHM $\tau_{\text {out }}=38.1 \mathrm{~ms}$ ). In the pulse of $33 \mathrm{~ms}$-width $\left(\tau_{\text {in }}^{-1}=30.5 \mathrm{~Hz}\right)$ the output pulse does not suffer distortion. However, an appreciable shape deformation in the output pulses may appear when shortening in time-domain the input pulses. Thus, in order to study the shape of the output pulses we compute the output pulse-width through $\sigma_{\text {out }}$, the standard deviation of the mean, which is the square root of the second-order moment
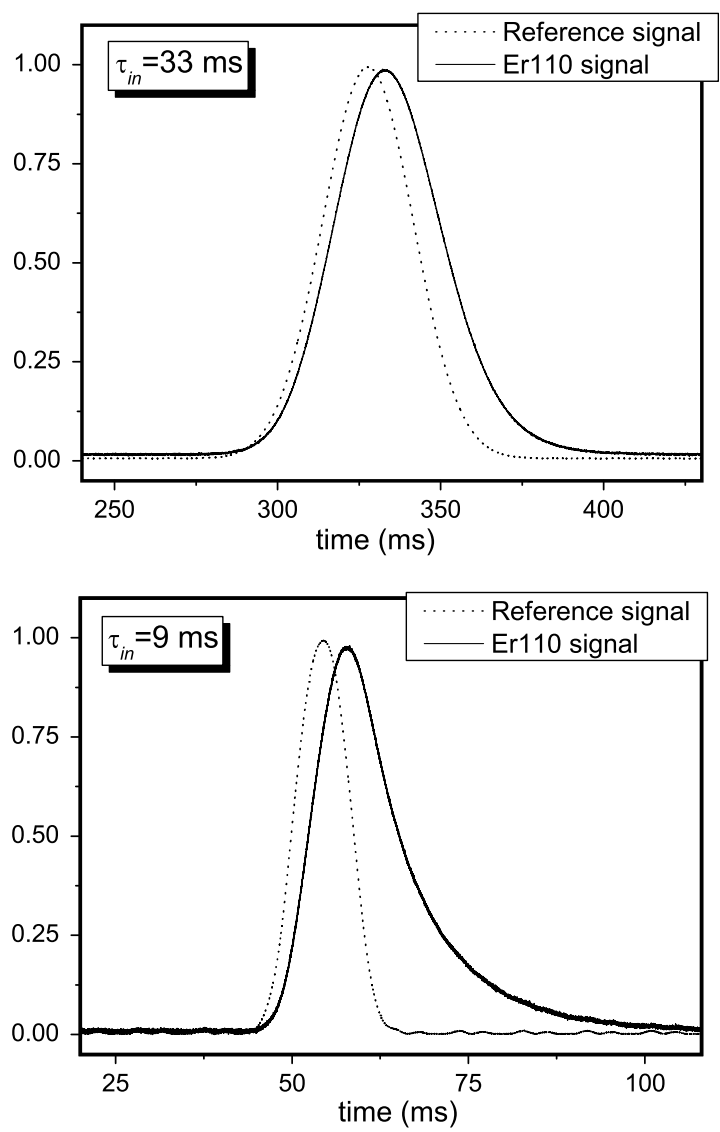

Fig. 9. Normalized Gaussian reference pulse (dotted line) and delayed pulse (solid line) after propagating through the Er20 fiber (left); and after propagating through the Er110 fiber (right). (Top) Input pulse-width $\tau_{\text {in }}=33 \mathrm{~ms}$. (Bottom) Input pulse-width $\tau_{\text {in }}=9 \mathrm{~ms}$. In all cases the laser input power was $P_{\mathrm{bg}}=0.06 \mathrm{~mW}$ and $P_{\text {pulse }} / P_{\mathrm{bg}}=25$ for the Er20 fiber and $P_{\text {pulse }} / P_{\mathrm{bg}}=29$ for the Er110 fiber. 
of the output pulse intensity distribution. In the previous example, the input pulse-width calculated using the variance of the distribution is $\sigma_{\mathrm{in}}=14 \mathrm{~ms}$ (which agrees with $\sigma_{\text {in }}=(2 \sqrt{2 \ln 2})^{-1} \tau_{\text {in }}$, while the output pulse-width after propagating through the Er20 and Er110 fiber are $\sigma_{\text {out }}=11.1 \mathrm{~ms}$ and $\sigma_{\text {out }}=17.1 \mathrm{~ms}$, respectively. Although, as we said above, the output pulse does not suffer an appreciable distortion (see Fig. 9 (top)), the relation between $\sigma_{\text {out }}$ and $\tau_{\text {out }}$ slightly deviates from the case of a perfect gaussian pulse.

In Fig. 9 (bottom) a short input pulse $\left(\tau_{\text {in }}=9 \mathrm{~ms}\right)$ is propagated through both fibers. As in the case of the propagation of modulated signals presented in previous section (Section 4.1), these high-bandwidth pulses will show larger fractional delays: $F=0.13$ for $\operatorname{Er} 20$ fiber and $F=0.57$ (i.e., a group velocity of $322 \mathrm{~m} / \mathrm{s}$ ) for Er110. Furthermore, this short pulse does not suffer any appreciable shape deformation when propagating through the Er20 fiber (see left part of Fig. 9 (bottom)). However, an appreciable broadening of the tail observed when propagating the same pulse through the ultra-highly doped fiber Er110 leads to $\sigma_{\text {out }}$ $=3.4 \sigma_{\text {in }}$ (see right part of Fig. 9 (bottom)). This deformation arises from the fact that the pulse bandwidth $\left(\tau_{\mathrm{in}}^{-1}=110 \mathrm{~Hz}\right)$ is larger than the linewidth of the CPO hole.

We have analyzed in detail the fractional delay and pulse broadening/compression for propagating pulses of different width along the erbium doped fibers. The results are summarized in Fig. 10 for a fixed pulse-to-background power ratio around 25. On the left of Fig. 10 we plotted the fractional delay as a function of the pulse-width $\tau_{\text {in }}$ for all fibers. As in the case of the propagation of modulated signals (Section 4.1), the most highly doped fibers exhibit the largest fractional delays. In fact, Er20 fiber shows a maximum fractional delay of $15 \%$ for input pulses of $\tau_{\text {in }}=10 \mathrm{~ms}$, whereas the maximum fractional delay achieved in the Er110 fiber is around $70 \%$ for input pulses of $\tau_{\text {in }}=2 \mathrm{~ms}$ (see inset in Fig. 10 (left)). We must point out that the maximum fractional delay occurs for pulse dura- tions close to $f_{\mathrm{opt}}^{-1}$. These results confirm that the ultra-high EDFs (Er80 and Er110) allow the propagation of pulses with higher bandwidths and larger fractional delays than those within the high doped fibers.

As we said before, the pulse width is not preserved during propagation along the fibers. In fact, depending upon both the duration of the pulse and the ion's concentration, we obtain pulse narrowing or pulse broadening. The quotient between the width of the output pulse, $\sigma_{\text {out }}$, and the width of the input pulse, $\sigma_{\text {in }}$, may serve as an indicator of pulse-width modifications. Fig. 10 (right portion) shows the results obtained for the different cases considered. Pulse broadening is always obtained in the case of ultra-highly doped fibers, i.e., $\sigma_{\text {out }} / \sigma_{\text {in }}>1$. However, in the highlydoped fibers (Er20-Er40) a transition from broadening to compression takes place. This transition takes place for pulse-widths of $\tau_{\text {in }} \approx 12 \mathrm{~ms}$ (on Er20), $16 \mathrm{~ms}$ (on Er30) and $22 \mathrm{~ms}$ (on Er40). These times are roughly the $f_{\mathrm{opt}}^{-1}$ obtained for the modulated signals when considering a laser input power of $P=P_{\mathrm{bg}}+P_{\text {pulse }} \simeq 1.6 \mathrm{~mW}$ (see Fig. 7). Thus, in the case of pulses lesser/larger in width than $f_{\text {opt }}^{-1}$, pulse broadening/narrowing is obtained.

This striking behavior can be explained in terms of two competing mechanisms [21]. The first consists of a timedependant saturation of the absorption. The leading edge of a high power pulse (above saturation) will saturate the absorption of the medium, allowing the trailing part to see increased transmission relative to the front. This leads to a compression of the pulse (see Basov effect [22,23]), provided the time duration of the pulse is greater than the absorption recovery time. This mechanism explains pulse compression in Er20, Er30 and Er40 fibers. However, the high concentration of erbium ions in the ultra-highly doped fibers prevents saturation, inhibiting the compression from taking place. Below saturation, the narrow hole created by CPO in the EDF absorption profile causes the wings of the pulse spectrum to experience a larger absorption than the central frequency components, thereby compressing
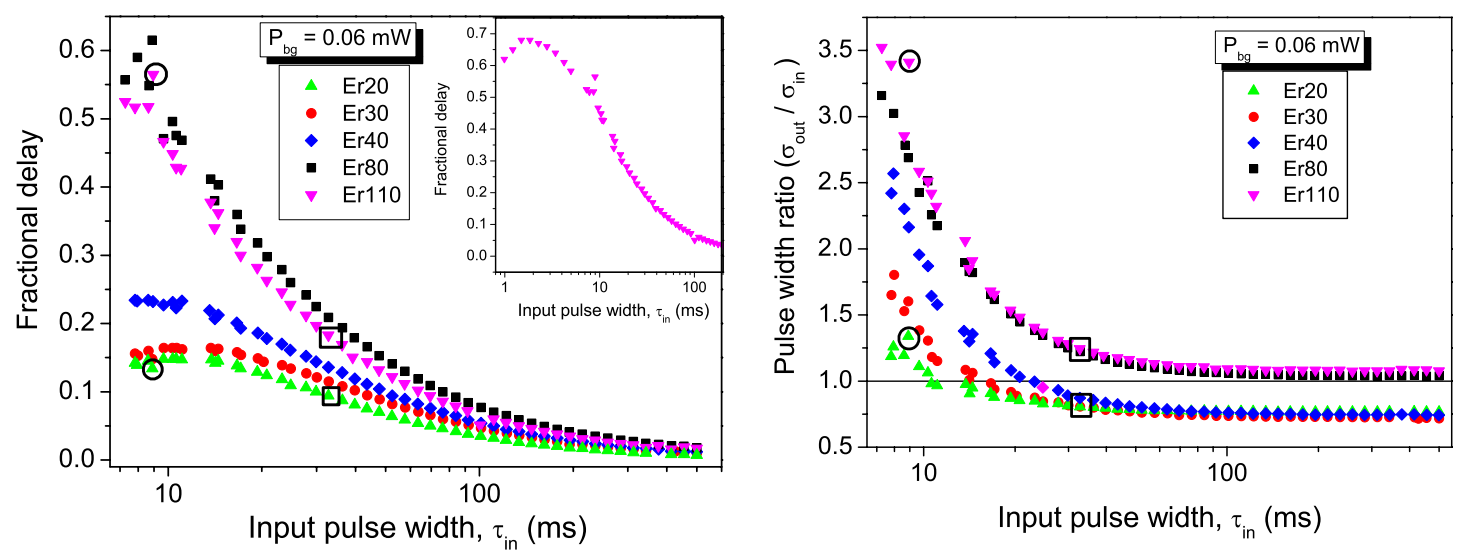

Fig. 10. Fractional delay (left) and pulse width ratio (right) vs. the input pulse width for all fibers in a semilog scale. In all cases the laser input power was $P_{\mathrm{bg}}=0.06 \mathrm{~mW}$ and the ratio between pulse and background power was around $P_{\text {pulse }} / P_{\mathrm{bg}} \approx 25$. In particular 25 (for Er20), 21 (for Er30), 25 (for Er40), 29 (for Er80), and 29 (Er110). Square open boxes refer to the particular cases plotted in Fig. 9 (top), while open circle symbols refer to the particular cases plotted in Fig. 9 (bottom). 
the pulse spectrum and broadening the pulse in time domain $[21,24]$. This mechanism explains pulse broadening in Er80 and Er110 fibers. The broadening of the pulse will be especially noticeable for short pulses when a significant part of the pulse does not lie within the hole in the absorption profile.

Finally, we have analyzed pulse distortion as a function of the pulse power. We used the Er20 fiber to be able to sweep power values from below to above the saturation power. In Fig. 11 (top) we plotted the pulse width ratio versus input pulse-width at different pulse-to-background power ratios: $P_{\text {pulse }} / P_{\mathrm{bg}}=6$ (circle), 12 (square), 25 (up triangle), 40 (down triangle), and 63 (rhombus). In those measurements laser input power was fixed to $P_{\mathrm{bg}}=0.06 \mathrm{~mW}$. We observed shortening or broadening of pulses, depending upon both pulse-width and pulse peak-power. The shown behavior can be understood considering the mechanisms previously mentioned. For sufficiently powerful pulses of $P_{\text {pulse }} / P_{\mathrm{bg}}=63$, the Basov effect dominates pulse distortion and, thus, compression of the pulse is obtained. When the peak pulse power decreases, larger temporal width is required to maintain the compression regime. Otherwise, pulse broadening dominates due to the spectrum compression created by CPO. In Fig. 11 (bottom) we show the experimentally measured
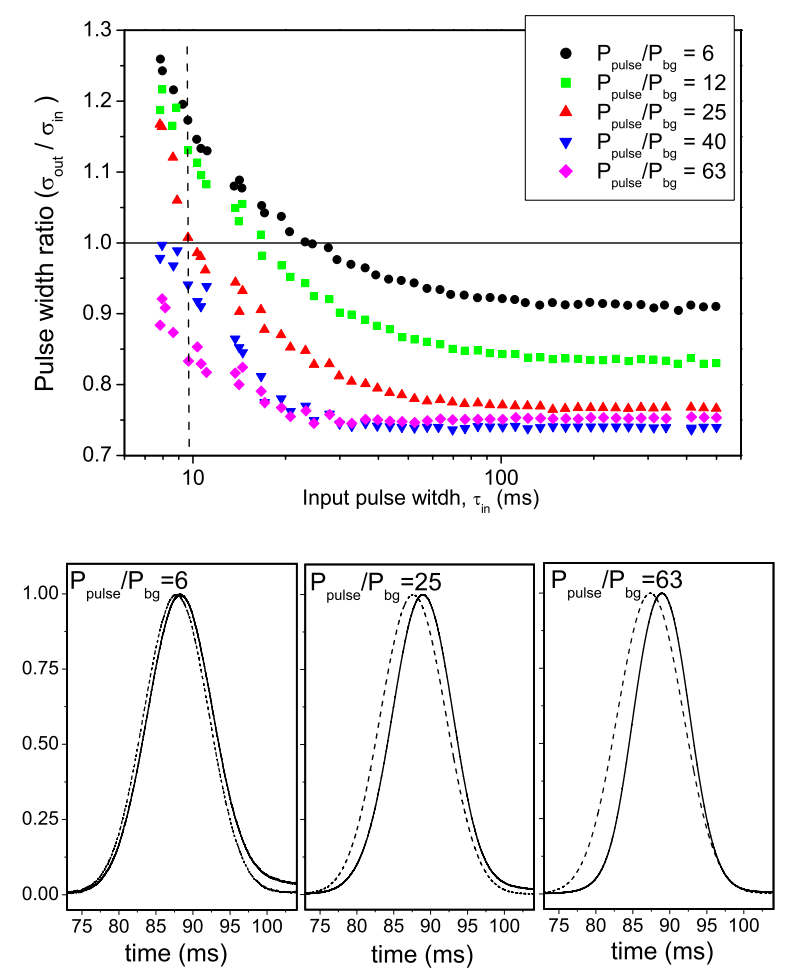

Fig. 11. (Top) Pulse width ratio versus input pulse width for the Er20 fiber and different pulse-to-background power ratios: $P_{\text {pulse }} / P_{\text {bg }}=6$ (circle), 12 (square), 25 (up triangle), 40 (down triangle), and 63 (rhombus). (Bottom) Experimentally measured input (dashed line) and output (solid line) pulse waveforms of 10-ms pulse-width at power ratios 6 (broadening), 25 (no pulse-width distortion), and 63 (compression). Optical fiber Er20 and laser input power $P_{\mathrm{bg}}=0.06 \mathrm{~mW}$. input pulse (dashed line) of $10 \mathrm{~ms}$ and the output pulse waveforms (solid line) at power ratios $P_{\text {pulse }} / P_{\mathrm{bg}}=6,25$, and 63. The pulse was propagated along the Er20 fiber and we used a laser input power of $P_{\mathrm{bg}}=0.06 \mathrm{~mW}$. The output pulse broadens or compresses depending upon the peak pulse power.

\section{Conclusions}

We have carried out experiments on slow-light propagation at room temperature in EDFs with different levels of ion doping (from $800 \mathrm{ppm}$ to $4350 \mathrm{ppm}$ ) in the range of wavelengths used in telecommunications. We measured the fractional delay of sinusoidally modulated signals as a function of the modulation frequency. For low input laser powers, highly doped fibers show a bandwidth close to $31 \mathrm{~Hz}$, whereas at extremely high doping levels the bandwidth is enhanced roughly by a factor of 5 . In addition the maximum fractional delay available changes from $4 \%$ for highly doped fibers to $18 \%$ for the ultra-highly doped fibers.

The influence of average power $P_{0}$ upon the maximum available fractional delay $F_{\max }$ has been also addressed. The experimental results illustrate that for highly doped fibers the curves of fractional delay versus modulation frequency are power-dependent. In fact, the modulation frequency at which the maximum fractional delay is achieved, the so-called optimum frequency, can be tuned from low values $(30 \mathrm{~Hz})$ to large values $(230 \mathrm{~Hz})$ by simply changing the value of $P_{0}$ but at the expense of reducing the maximum fractional delay. However, for ultra-highly doped fibers, the optimum frequency is obtained for low values of $P_{0}$ with the additional advantage of providing a large bandwidth. Thus we may conclude that ultra-highly doped fibers provide larger bandwidth, larger fractional delay, and lower power consumption than moderate and highly doped fibers.

We have also analyzed how finite-band signals propagate through the fibers. The pulses were Gaussian in shape and superimposed over a DC component of power $P_{\mathrm{bg}}$. Fractional delays of up to $70 \%$ have been measured for millisecond pulses with the highest doping levels used. Finally, distortion of the propagated pulses has been analyzed for different fibers. We observed both compression and broadening of the propagated pulses for the leastdoped fibers used in the experiments. However, only broadening of the pulses has been measured for fibers doped above $3150 \mathrm{ppm}$. We found that pulse distortion can be avoided by controlling the peak pulse power for each broadband-pulse.

\section{Acknowledgements}

This work has been supported by Projects No. FIS200403267 (MEC, Spain), PR45/05-14183 (UCM/CM, Spain), PR27/05-14019 (UCM/BSCH, Spain), AE5/06-14369 (UCM, Spain), and CCG06-UCM/ESP-1317(UCM, 
Spain) S.M. and O.G.C. thank R.W. Boyd, M.S. Bigelow, E. Baldit, A. Schweinsberg, M. González Herráez, J.M. Guerra, R. Weigand, I. Gonzalo, and J.M. Rico for helpful advice. We are grateful to T. Lorca and C.E. Caro for the English revisions of the manuscript.

\section{References}

[1] L.W. Hillman, R.W. Boyd, J. Kransinski, C.R. Stroud Jr., Opt. Commun. 45 (1983) 416.

[2] S.E. Harris, J.E. Field, A. Kasapi, Phys. Rev. A 46 (1992) R29.

[3] A. Kasapi, M. Jain, G.Y. Yin, S.E. Harris, Phys. Rev. Lett. 74 (1995) 2447.

[4] L.V. Hau, S.E. Harris, Z. Dutton, C.H. Behroozi, Nature (London) 397 (1999) 594.

[5] M.M. Kash, V.A. Sautenkov, A.S. Zibrov, L. Hollberg, G.R. Welch, M.D. Lukin, Y. Rostovtsev, E.S. Fry, M.O. Scully, Phys. Rev. Lett. 82 (1999) 5229.

[6] D. Budker, D.F. Kimball, S.M. Rochester, V.V. Yashchuk, Phys Rev. Lett. 83 (1999) 1767.

[7] A.V. Turukhin, V.S. Sudarshanam, M.S. Shahriar, Phys. Rev. Lett. 88 (2002) 023602.

[8] M.S. Bigelow, N.N. Lepeshkin, R.W. Boyd, Phys. Rev. Lett. 90 (2003) 113903

[9] M.S. Bigelow, N.N. Lepeshkin, R.W. Boyd, Science 301 (2003) 200.
[10] P.C. Ku, F. Sedgwick, C.J. Chang-Hasnain, P. Palinginis, T. Li, H. Wang, S.W. Chang, S.L. Chuang, Opt. Lett. 29 (2004) 2291.

[11] X. Zhao, P. Palinguinis, B. Pesala, C.J. Chang-Hasnain, P. Hemmer, Opt. Express 13 (2005) 7899.

[12] H. Su, S.L. Chuang, Opt. Lett. 31 (2006) 271.

[13] E. Baldit, K. Bencheikh, P. Monnier, J.A. Levenson, V. Rouget, Phys. Rev. Lett. 95 (2005) 143601.

[14] A. Schweinsberg, N.N. Lepeshkin, M.S. Bigelow, R.W. Boyd, S. Jarabo, Europhys. Lett. 73 (2006) 218.

[15] R.W. Boyd, D.J. Gauthier, A.L. Gaeta, Opt. Photon. News 17 (2006) 18.

[16] P.F. Wysocki, J.L. Wagener, M.J.F. Digonnet, H.J. Shaw, Proc. SPIE Fiber Laser Sources Amplifiers IV 1789 (1992) 66.

[17] E. Snoeks, G.N. van den Hoven, A. Polman, B. Hendriksen, M.B. Diemeer, F. Priolo, J. Opt. Soc. Am. B 12 (1995) 1468.

[18] Y.L. Xue, G.B. Smith, A.T. Baker, IEEE J. Quantum Electron. 34 (1998) 1380

[19] M.V.D. Vermelho, U. Peschel, J.S. Aitchison, J. Lightwave Technol. $18(2000) 401$.

[20] B-Ch. Hwang, S. Jiang, T. Luo, J. Watson, G. Sorbello, N. Peyghambarian, J. Opt. Soc. Am. B 17 (2000) 833.

[21] H. Shin, A. Schweinsberg, G. Gehring, K. Schwertz, H.J. Chang, R.W. Boyd, Q.-H. Park, D.J. Gauthier, Opt. Lett. Doc. ID 78405 (2007).

[22] N.G. Basov, R.V. Ambartsumyan, V.S. Zuev, P.G. Kryukov, V.S. Letokhov, Sov. Phys. JETP 23 (1966) 16.

[23] A.C. Selden, Brit. J. Appl. Phys. 18 (1967) 743.

[24] H. Cao, A. Dogariu, L.J. Wang, IEEE J. Sel. Top. Quantum Electron. 9 (2003) 52. 